\title{
Whisker's Directional Selectivity: Orientation Columns in the Barrel Field?
}

\section{Keywords: optical imaging; voltage-sensitive dye; barrel field; rat; somatosensory cortex; whiskers; angular selectivity}

\section{INTRODUCTION}

Within the past century neuroscientists have discovered many functional maps in the mammalian brain, but until recently, data had led the scientists to assume that detailed maps of directional sensory information from the outside world were located only in the visual cortex (Imamura et al, 2006). Decades ago the barrel-shaped cortical structures known as the barrel field were found to map each whisker in a precise location. Recent studies (Andermann and Moore, 2006; Andermann and Moore, 2008; Boas eat al, 2008 Diamond et al., 2008; Frostig, 2006; Li and Ebner, 2007; Timofeeva et al., 2003) have discovered that the barrel cortex also contains a map of directional sensitivity. This new map of directional sensitivity has, until now, been studied only using electrophysiological methods (Andermann and Moore, 2006; Borgdorff et al, 2007) which, while very sensitive in time, lack spatial precision for localizing brain activity. Our research group is currently using voltage-sensitive dye optical imaging methods in order to create a functional map of the directional sensitivity of the barrel field. This technique has yielded revolutionary results when applied to the visual (Tanaka et al., 2009), auditory (Song et al, 2006; Tsytsarev et al., 2009) and somatosensory (Devor at al., 2005; Civillico and Contreras, 2006; Chen-Bee at al, 2007) systems. Optical imaging based on voltage-sensitive dye signals allows us to make a functional map of the 
barrel field. Here, we demonstrate that spatially organized neurons in the somatosensory cortex show preference for certain directions of whisker deflection.

Many studies, especially of sensory cortices, have investigated information processing within and between these sub-regions of the cortex (Dehnardt et al, 1999;

Derdikman et al, 2003; Haidarliu and Ahissar, 2001; Peterseb and Sakmann, 2001). Because of the unique relationship between individual facial vibrissae and corresponding barrels of the rat neocortex, the barrel field has been particularly useful as a model system for studying the relationship between how the cortex is organized and how it processes sensory information (Feldman and Brecht, 2005; Ferezou et al, 2006; Civillico and Contreras, 2006; Narumi et al, 2007; Petersen, 2003; Petersen et al, 2003). As we can see in the obtained data, the voltage-sensitive dye optical signal has a different spatial representation in response to different directions of single whisker deflection. We believe this suggests that the barrel has directionally - preferred neurons that correspond to the direction of whisker deflection based on inputs from the cells of the whisker pad.

\section{MATERIALS AND METHODS}

Imaging of voltage-sensitive dye optic signals was performed on 6 adult rats (Sprague-Dawley, male, weight 200-300 g; age 2-3 months). All animals were initially anesthetized with intraperitoneal ketamine ( $90 \mathrm{mg} / \mathrm{kg}$, Hospira) and xylazine $(2.0 \mathrm{mg} / \mathrm{kg}$ body weight, i.m., Hospira) and then sustained with urethane $(1.25 \mathrm{gm} / \mathrm{kg})$, and furosemidae sulfate $(0.1 \mathrm{mg} / \mathrm{kg}$ body weight, i.m., Hospira) (Tsytsarev et al, 2008). Body temperature was maintained at $37^{\circ} \mathrm{C}$ with a heating blanket (Harvard Apparatus, Holliston, MA). 
The animal was rigidly fixed in a stereotaxic frame placed on a vibroisolated table (Bahar et al, 2006). The skin at the dorsal part of the head was removed so that the cranium above the left somatosensory cortex could be removed using a dental drill. A chamber made of high density silicon was constructed above the hole in the skull and dura mater was removed at the recording area. A detailed description of the experimental technique has been described previously (Tsytsarev, 2008). The voltage-sensitive dye, RH-1691 (Optical Imaging, $0.6 \mathrm{mg} / \mathrm{ml}$ in artificial cerebrospinal fluid (ACSF)), was applied to the exposed cortex for approximately $1 \mathrm{hr}$. After staining, the cortex was washed with dye-free ACSF for approximately $15 \mathrm{~min}$. To suppress brain pulsation originating from respiratory and cardiovascular movements, the chamber was filled with agar ( $0.3 \%$ in ACSF) and then sealed with a cover. A 16-bit CCD camera (Cascade 512B, Roper Scientific) was positioned above the chamber and directed such that its optical axis was perpendicular to the cortical surface and focused $0.3 \mathrm{~mm}$ below the cortical surface.

A tungsten filament lamp (12 V, 100W, Zeiss) was used for illumination of the cortex. The light passed through a heat filter (Zeiss), a $630 \pm 20 \mathrm{~nm}$ interference filter (Edmund Optics), and a dichroic mirror (650DRLP, Optical Imaging Inc). Imaging was performed through a tandem configuration of two $50 \mathrm{~mm}$ lenses, at a frame rate of $10 \mathrm{~ms}$ / frame and recorded using Metamorph software (Molecular Devices) on a Windows XP platform. Experiments consisted of 200 trials of stimulation, one trial per stimulus. Change in fluorescence was calculated as $\Delta \mathrm{F} / \mathrm{F}(\%)$ in the region of interest using custom-written software on the Matlab (The Mathworks) platform. The recording trials consisted of 400 frames, with the stimulus being presented at the $200^{\text {th }}$ frame. 
The final three prestimulus frames were averaged and used as a baseline, subtracted from each subsequent frame to decrease noise (Tsytsarev et al., 2004). This procedure (firstframe analysis) was applied to the recorded signals at each trial, which were then averaged. Pseudocolor images (Figure 1, A) of the cortical surface were generated after the experiments (Chen-Bee et al, 2007).

The algorithm of each experiment is as follows: TTL-pulse is generated by the Spike2 data acquisition system (Cambridge Electronic Design, 2001) every 40 seconds. This TTLpulse starts a recording trial which contains 400 frames of $10 \mathrm{~ms}$ duration each. Each trial forms a single record file. The multidirectional mechanical whisker stimulator acts as a step relay. Each impulse activates the deflection of vibrissae in one of four directions: impulse 1 causes a movement backward (stimulus 1); 2, upward (stimulus 2); 3, downward (stimulus 3); 4, forward (stimulus 4); 5, again backward and etc. To avoid tinkling, the whisker stimulation pulse for whisker stimulation was $15 \mathrm{~ms}$ long and had a trapezoidal shape with both slopes being $5 \mathrm{~ms}$ in duration. The deflection of vibrissae starts during the $200^{\text {th }}$ frame. The inter-trial interval was 40 seconds.

A part (2-3 $\mathrm{mm}$ ) of the vibrissa was covered with a suspension of iron powder (Sigma Aldrich, \# 12311) in Plexiglas, saluted in acetone. Deflection was performed using a custombuilt deflecting device with four small crosswise electromagnets; the whisker was placed at the intersection of the magnet axes. In each experiment the single vibrissae E2 was deflected in four directions using ramp-and-hold stimuli delivered through an in-house designed laboratory electromagnetic stimulator. The whisker was moved from its neutral (central) position in different directions. The stimulator was attached to the whisker $4-5 \mathrm{~mm}$ from the skin surface to produce deflection amplitudes of $0.2-0.3 \mathrm{~mm}$. After the termination of 
the experiment the animal was sacrificed by injection of $1.0 \mathrm{ml}$ of Sleepaway (Hospira, 2006) and its brain was removed.

To distinguish the source of the optic signal morphologically, we superimposed the imaging data with histological data. We used a cytochrome oxidase (CO)-stained section in layer IV of the flattened left hemisphere of three animals to see the barrel field and compare the VSD data and morphological structure of the barrel field (Kerr at al., 2007; Shimegi et al, 2000; Timofeeva, 2003 ). The brain was stained with $0.1 \mathrm{M}$ phosphate buffer followed by paraformaldehyde fixative ( $\mathrm{pH}$ 7.4). To obtain a complete section of layer IV, the naturally curved cortex was flattened (Welker and Woolsey, 1974) and immersed in a 30\% sucrose buffer solution for $48 \mathrm{~h}$. Sections were cut tangential with a cryotome at a thickness of $50 \mu \mathrm{m}$ and were incubated at $37^{\circ} \mathrm{C}$ in the dark for $1 \mathrm{~h}$. The incubation medium was prepared immediately before use. It consisted of $10 \mathrm{ml} 0.1 \mathrm{M}$ phosphate buffer, $\mathrm{pH} 7.4,100 \mu 1$ diamoinobenzidine $(25 \mathrm{mg} / \mathrm{ml}), 0.002 \mathrm{~g}$ cytochrome c, $0.5 \mathrm{~g}$ sucrose, 2 drops $1 \%$ Nickel ammonium sulfate, 2 drops Triton X-100, and some catalase. Sections were rinsed in three changes of $0.1 \mathrm{M}$ phosphate buffer, mounted, air dried, dehydrated and coverslipped. Pseudocolor images obtained directly by VSD optical imaging were superimposed on photographs of cytochrome oxidase stained sections to map optical signal matched barrels of the stimulated vibrissa (Figure 1, B).

For initial optical imaging, we constructed a functional map using first frame analysis as defined above for each individual trial (Tsytsarev et al, 2008) and then averaging the data for each stimulus. As a result we received an image of the activated area in each frame as a response to each stimulus (Fig. 2, A). For the spatio-angular analysis we used an optical signal that had been obtained before and after the impact of the stimulus. At this point in the 
analysis and throughout the rest of the study we used the pixels of the CCD-camera as a scale factor, since the area of recording was always 40 x 40 pixels. All other parameters of recording were also kept constant.

After first frame analysis, the activated areas were identified by the number of activated pixels exhibiting a change in fluorescence $(\Delta \mathrm{F} / \mathrm{F})$ greater than $0.5 \%$ (fixed threshold). We applied this fixed threshold for all the data collected in the 6 control experiments (4 stimuli each), and thus determined areas (activity patterns) activated by different stimuli (Fig 2, B). Then, using corresponding MATLAB code, we found the center of mass $\mathrm{C}_{i}\left(\mathrm{x}_{i}, \mathrm{y}_{i}\right)$ of the activated pattern for each stimulus $i(i=1,2,3,4)$ for each experiment (see in Figure 2, B), and calculated the average center of mass $\mathrm{C}_{j}\left(\mathrm{x}_{j}, \mathrm{y}_{j}\right)$ for each stimulus $(\mathrm{j})$ :

$$
x_{j}=\frac{x_{1}+x_{2}+x_{3}+x_{4}}{4} ; \quad y_{j}=\frac{y_{1}+y_{2}+y_{3}+y_{4}}{4}
$$

An example for one particular experiment is shown in Fig. 2C. In order to standardize all experiments to common coordinates we found the common center of mass of all stimuli in all experiments by superposition of partial centers $\mathrm{Cj}$. The point of origin in a common frame of reference we designated as $\mathbf{O}(0,0)$.

For each experiment we calculated the angles $\alpha_{n m}=C_{n} \mathbf{O C}$, where $C_{n}$ is the center of mass of the pattern activated by stimulus $n$, and $C_{m}$ is the center of mass of the pattern activated by stimulus m: 


\section{$\alpha_{n m}=\operatorname{arctg}\left(\frac{y_{n}}{x_{n}}\right)-\operatorname{arctg}\left(\frac{y_{m}}{x_{m}}\right)$}

The calculated values of the angles were then mapped onto the coordinate axis by choosing the smallest of conjugate angles (e.g., an angle of 200 degrees is complimented to 160 degrees, see figure). Finally, we obtained the angles between directional activations by aligning the centers of mass of the patterns activated by all stimuli in all experiments to the common center of mass.

\section{RESULTS}

For all eight examined rats voltage-sensitive dye optical signals elicited in response to directional whisker stimulation were observed in a cortical area anatomically corresponding to the barrel field of the left hemisphere. The activated patterns, shown in Figure 1, A, were derived from activated areas exhibiting a change in fluorescence $(\Delta \mathrm{F} / \mathrm{F})$ greater than $0.5 \%$. The choice of a $0.5 \%$ threshold was motivated by the fact that this is approximately half the dynamic range of the fluorescence signal (Tsytsarev et al, 2008; Tsytsarev et al, 2009). Time courses of the fluorescence signals obtained in response to all stimuli in all experiments were averaged and are shown in Fig.1, B. We have not observed a significant difference between the optical time courses in response to different stimuli.

The number of pixels where the optical signal was in excess of threshold was analyzed statistically (Fig. 3B). The signal usually appeared just after the frame when the 
stimulus was presented and reached its peak 20 - 30 ms after stimulus onset. After reaching their maximum value the activated areas decreased until returning to baseline $20-40 \mathrm{~ms}$ after the stimulus onset. All these properties of directional representation could be reproduced in all rats examined. We did not observe a significant distinction between temporal features of signals in response to different directions of the whisker's deflection.

The most interesting obtained data are relevant to the spatial structures of the optical response. The angles created by drawing a line from one activated pattern's center of mass to the common center of mass and then to a second activated pattern's center of mass have been calculated for all pairs of stimuli in all experiments. These centers of mass were then mapped onto a single coordinate system. The estimated values for those angles were analyzed statistically.

If the angle between two directions in which the whisker was deflected was 90 degrees (using the common center as an anchor as defined above), the angle between the directions from the common center of mass and the centers of mass of corresponding activity patterns does not significantly differ from 90 degrees in three cases of four (2-tales Z-test, $\mathrm{n}=6 ; \mathrm{P}=0.5315$ for angles between stimulus 1 and $2, \mathrm{P}=0.0763$ for stimuli 2 and 3 and $\mathrm{P}=0.6389$ for stimuli 1 and 4). In one case (stimuli 3 and 4) the difference from 90 degrees was statistically significant (2-tales $\mathrm{Z}$-test, $\mathrm{n}=6, \mathrm{P}=0.03474$ ). Similarly, the angle formed from mapping activations between stimulation directions separated by 180 degrees was standardly close to 180 degrees ( $\mathrm{P}=0.8237$ for stimuli 1 and $3, \mathrm{P}=0.8589$ for 2 and 4 .) These results are shown in Fig. 3-C.

Thus, it is possible to separate the responses reliably into two groups based on the spatial characteristics of the stimulus. The angles of the first group (stm1-0-stm3 and stm2- 
0 -stm4) are close to 180 degrees whereas the angles of the second group (stm1-0-stm2 and stm3-0-stm4) are close to 90 degrees. This is an important result: the angles between directions of whisker deflection are also 180 degrees for whisker stimuli 1 / $3(\mathrm{P}=0.8237)$ and stimuli 2 / 4 ( $\mathrm{P}=0.8589)$, and 90 degrees -between stimuli 1-2 $(\mathrm{P}=0.5315), 3-4(\mathrm{P}=0.0347$; not significantly), 2-3 $(\mathrm{P}=0.0763)$ and $1-4(\mathrm{P}=0.6389)$. All centers of mass (all stimuli - all experiments) plotted in uniform co-ordinates are shown in Fig 3A.

\section{DISCUSSION}

The whisker system is critical to rodents' ability to obtain information about the world around them (Stanley, 2009). Thus it is a logical inference that the whisker's angular selectivity is a key element of barrel cortex stimulus representations. Angular selectivity of the neurons has been described at different levels of the vibrissae system (Li, L; Ebner F., 2007; Minnery BS., Simons DJ. , 2003; Hemelt, M; Keller; 2007) but it's mechanism is still not clarified completely.

Anatomically, sensory information comes from the follicle/ whisker complex to the cortex via three different pathways (Minnery and Simons 2003; Kwegyir-Afful and Keller, 2004). In the lemniscal pathway, second order neurons in the trigeminal nuclei (TN) are clustered into "barrelettes". Their axons travel to the opposite side of the brain to the "barreloids" of the dorsomedial section of the ventral posterior medial nucleus (VPMdm) of the thalamus. Both barrelettes and barreloids are sets of modules arranged as a topographic projection of the whiskers. The axons of VPMdm neurons project to the barrels of the primary somatosensory cortex (S1). 
In the extralemniscal pathway neurons in the caudal part of the $\mathrm{TN}$ are also clustered into whisker-related barrelettes. They project to the ventrolateral domain of the ventral posterior medial nucleus (VPMvl), where neurons are clustered into the barreloids of the VPMdm. The axons of VPMvl neurons project to the S1 and secondary somatosensory cortex (S2). However, the neurons of the paralemniscal pathway in the rostral part of the TN are not spatially clustered. They project to the medial sector of the posterior nucleus (POm). The axons of POm neurons project to targets immediately ventral to the S1 and S2 (Fig. 3, D).

Accordingly, neurons of the lemniscal pathway of the rat have the highest angular selectivity of the pathways (Minnery and Simons 2003). To determine if this property also characterizes neurons of the secondary somatosensory cortex, Kwegyir-Afful and Keller (Kwegyir-Afful and Keller, 2004) recorded responses to whisker deflections in eight different directions. According to their results the secondary somatosensory cortex contains a large number of neurons with strong angular selectivity, and they are more selective than the neurons of the barrel for the angle of whisker deflection. Subcortical neurons demonstrate relatively strong angular preference. This probably reflects the configuration of the receptors at the base of the whisker follicle (Andermann at al, 2008; Boas et al, 2008). But, the number of well -tuned neurons decreases at subsequent levels of the pathway between the whisker and the barrel: in trigeminal ganglion $81 \%$ of the neurons are well tuned (Kwegyir-Afful EE and Keller, 2004), 31.1\% in VPm, and only 15.7\% in the barrel field (Kwegyir-Afful EE and Keller 2004 , Minnery and Simons, 2003). We agree with the hypothesis of Kwegyir-Afful EE and Keller that this progressive decrease may be due to convergence of inputs from neurons with different and sometimes opposing angular preferences. 
According to the results of numerous studies (Andermann and Moore, 2006 and 2008; Batuev at al., 1989; Furuta et al., 2009; Khatri and Simons, 2006), neurons of the rat barrel field are sensitive to the angular direction of whisker movement. We have used voltage-sensitive dye imaging in combination with computational methods to analyze the microanatomical representation of angular selectivity. Our obtained optical imaging data correspond to electrophysiological data obtained previously by many authors (and Moore, 2006 and 2008; Batuev at al., 1989). These results also suggest that directional selectivity has a clear vertical organization in the barrel field of the somatosensory cortex. The strong compliance between the arrangement of the centers of mass of the patterns activated by different stimuli, and disposition of these stimuli permits us to draw a conclusion about the functional representation of angular selectivity in the somatosensory cortex. We suppose that the angular-sensitive neurons are situated and organized in a circular formation around the barrel.

It is likely that angularly selective neurons in the barrel are characterized by a Vshaped histogram of deflection angle / deflection intensity similar to how the neurons in the auditory cortex have a V-shaped histogram of sound frequency / sound intensity. Each neuron is most sensitive to vibrissa deflection in one strictly defined direction (Fig. 4). Increase of the deflection's strength is indicated by an increase of the number of neurons which respond to the deflection. Presence of these neurons and their morphological localization in a circular formation around the barrel is the key finding of our investigation.

In summarizing the obtained data, we suppose that barrel cortex neurons not only are selective for the angle of whisker deflection but also localized in the single barrel in accordance to their angular selectivity. Previously, Hemelt and Keller (Hemelt, M; Keller, 
2007) have reported that the neurons of the superior colliculus have more angular selectivity when compared to neurons in of barrel: $16 \%$ of barrel cortex neurons have a high selectivity index in comparison to 43 percent of collicular neurons. Thus, it is truly significant to see such a clear spatial representation of the angular electivity in the barrel area.

\section{ACKNOWLEDGMENTS}

We thank Dr. Sonya Bahar for providing very useful discussions and help in manuscript writing. The programs used for data analysis and to control equipment during experiments were written by Daisuke Takeshita, and authors thank him very much. 


\section{REFERENCES}

1. Andermann ML, Moore CI.A somatotopic map of vibrissa motion direction within a barrel column. Nat. Neurosci. 9(4):543-51, 2006

2. Andermann ML, Moore CI. Mechanical resonance enhances the sensitivity of the vibrissa sensory system to near-threshold stimuli. Brain Res. 15;1235 : 74-81, 2008

3. Boas DA, Jones SR, Devor A, Huppert TJ, Dale AM., Athinoula A. A vascular anatomical network model of the spatio-temporal response to brain activation. Neuroimage. 40(3):1116-29, 2008.

4. Batuev AS, Alexandrov AA, Shcheynikov NA, Kcharazia VN, and Chan CA. The role of inhibitory processes in the formation of functional properties of neurons in vibrissal projection zone of the cat somatosensory cortex; Brain Res 76:198-206, 1989

5. Bahar S, Suh M, Zhao M, Schwartz TH. Intrinsic optical signal imaging of neocortical seizures: the 'epileptic dip'. NeuroReport. 17(5):499-503, 2006.

6. Borgdorff A., Poulet J, Petersen C. Facilitating sensory responses in developing mouse somatosensory barrel cortex. J. Neurophysiol. 97:2992-3003, 2007. 
7. Civillico EF, Contreras D. Integration of evoked responses in supragranular cortex studied with optical recordings in vivo. J. Neurophysiol. 96(1):336-51, 2006.

8. Chen-Bee CH, Agoncillo T, Xiong Y, Frostig RD. The triphasic intrinsic signal: implications for functional imaging. J. Neurosci. 27(17):4572-86, 2007.

9. Devor A., Ulbert I., Dunn AK., Narayanan SN., Jones SR., Andermann ML., Boas D., Dale A. Neuronal basis of optical imaging signals in sensory cortex. Journal of Cerebral Blood Flow and Metabolism 25 (S683), 2005.

10. Diamond ME, Heimendahl M, Knutsen PM, Kleinfeld D, Ahissar E. 'Where' and 'what' in the whisker sensorimotor system Nat Rev Neurosci.9(8):601-612; 2008

11. Furuta,T., KanekoT., Desche, M. Septal Neurons in Barrel Cortex Derive Their Receptive Field Input from the Lemniscal Pathway The Journal of Neuroscience 29(13):4089-95; 2009

12. Hemelt, M; Keller; Superior sensation: superior colliculus participation in rat vibrissa system. BMC Neurosci. 8:12, 2007

13. Inyushin, M. Y., Volnova, A. B., and Lenkov, D. N. Use of a simplified method of optical recording to identify foci of maximal neuron activity in the somatosensory cortex of white rats. Neurosci. Behav. Physiol. 31, 201-205, 2001 
14. Imamura K., Tanaka S., Ribot J., Kobayashi M., Yamamoto M., Nakadate K., Watanabe Y. Preservation of functional architecture in visual cortex of cats with experimentally induced hydrocephalus. Eur J Neurosci. 23(8):2087-98, 2006.

15. Khatri V, Simons DJ. Angularly nonspecific response suppression in rat barrel cortex. Cereb Cortex. 2007;17(3):599-609.2006

16. Kerr JN, de Kock CP, Greenberg DS, Bruno RM, Sakmann B, Helmchen F. Spatial organization of neuronal population responses in layer $2 / 3$ of rat barrel cortex. J Neurosci. 2007 28;27(48):13316-28.

17. Kwegyir-Afful E.E., Keller A J Response Properties of Whisker-Related Neurons in Rat Second Somatosensory Cortex Neurophysiol 92: 2083-2092, 2004.

18. Li, L., Ebner F. Cortical Modulation of Spatial and Angular Tuning Maps in the Rat Thalamus. J of Neuroscience 27(1):167-179; 2007

19. Minnery B.S., Simons DJ. Response Properties of Whisker-Associated Trigeminothalamic Neurons in Rat Nucleus Principalis J Neurophysiol 89: 40-56, 2003. 
20. Petersen CH. Functional Organization of the Barrel Cortex. Neuron; 09(017) 339$354 ; 2007$

21. Shimegi S., Akasaki T., Ichikawa T., Sato H. Physiological and anatomical organization of multiwhisker response interactions in the barrel cortex of rats. J Neurosci. 20(16):6241-8., 2000

22. Stanley G.B. It's not you, it's me. Really. Nature neurosci, 12: 4, 2009.

23. Song W.J., Kawaguchi H., Totoki S., Inoue Y., Katura T.,Maeda S., Inagaki S., Shirasawa H., Nishimura M. Cortical intrinsic circuits can support activity propagation through an isofrequency strip of the guinea pig primary auditory cortex.Cerebral Cortex May 2006;16:718—729, 2005.

24. Tanaka S, Tani T, Ribot J, O'Hashi K, Imamura K. A postnatal critical period for orientation plasticity in the cat visual cortex. PLoS ONE. 2009;4(4):e5380

25. Timofeeva E., Merette C., Emond C., Lavallee P., Deschenes M. A Map of Angular Tuning Preference in Thalamic Barreloids. 23(33):10717-10723, J of Neuroscience, 2003 
26. Tsytsarev V, Premachandra K, Takeshita D, Bahar S. Imaging cortical electrical stimulation in vivo: fast intrinsic optical signal versus voltage-sensitive dyes. Optics Letters, (33) : 9, 1032-1034, 2008

27. Tsytsarev V., Fukuyama H., Pope D., Pumbo E., and Kimura M. (2009) Optical imaging of interaural time difference representation in rat auditory cortex; Frontiers in Neuroengineering; $2,1-7$

28. Welker C, Woolsey TA. Structure of layer IV in the somatosensory neocortex of the rat: description and comparison with the mouse. J Comp Neurol 15;158(4):437-53, 1974 
Figure 1

A. Voltage-sensitive dye optical images show single-whisker stimulation fluorescence changes. The stimulus onset was at the beginning of frame number one. Frame number indicated at the left bottom corner of each image; frame duration is $10 \mathrm{~ms}$. B.

Barrel field, its location in the rat's brain, and voltage-sensitive dye optical response in reply to whisker stimulation. From left to right: a horizontal slice of the rat somatosensory cortex from the barrel field's area stained for cytochrome oxidase; area of the craniotomy of the rat's brain postmortem; cortical surface prepared for voltage sensitive dye optical recording; barrel field's scheme. The same area is marked by blue and yellow squares. Right: change in fluorescence $(\Delta \mathrm{F} / \mathrm{F} \%$, ordinate) in response to different directional stimuli (denoted at the bottom left corner of the chart). Fluorescence signal was recorded in the small red rectangle marked at the cortical surface. Stimulus presented at the frame $200^{\text {th }}$ (abscissa)

Figure 2

Analysis of the obtained data. A. Colorized images of the voltage-sensitive dye optical signal combined with photography of the cortical surface. The stimuli number (direction of whisker's deflection) is shown in the left bottom corner of each image. B. The boundary of the areas activated by different stimuli, and their centers mass on the cortical surface. Stimuli adjustment is shown at the bottom. C. Centers of mass of the representation of four different stimuli and the common center (point of origin) in the co-ordinates. 


\section{Figure 3}

A. The centers of mass of the activity patterns plotted in a single coordinate system.

The stimulus number is denoted by color and the number of experiment by symbol.

B. Latency of the voltage-sensitive dye optical signal in response to various stimuli. Activity patterns depending on time represented by frame number. Duration of frame is $10 \mathrm{~ms}$. Frame number 200 is stimulus onset. C. Averaged angles between directions to the common center of mass and centers of mass of the different stimuli $(n=6)$. The stimuli 1-2, 2-3, 3-4 and 1-4 form right angles. Stimuli 1-3 and 2-4 form 180 degree angles. D. Sensory pathway of the vibrissa system. S1 - primary somatosensory cortex (barrel field); S2 - secondary somatosensory cortex; VPMdm - ventral posterior medial nucleus of the thalamus; VPMvl ventrolateral domain of the ventral posterior medial nucleus of the thalamus; TG trigeminal ganglion; $\mathbf{T N}$ - trigeminal nuclei. Projection of the optical signals obtained in response to different stimuli shown by different colors. 
